\title{
A Model for Mammogram Image Segmentation based on Hybrid Enhancement
}

\author{
Kamal Nain Sharma ${ }^{1}$, Amit Kamra ${ }^{2}$
}

Section: Healthcare

Sci. Journal Impact

Factor: 6.1 (2018)

ICV: 90.90 (2018)

'Research Scholar, IKGPTU, Kapurthala(PB), India; ${ }^{2}$ Assistant Professor, Guru Nanak Dev Engineering College, Ludhiana (PB), India.

\section{ABSTRACT}

Introduction: Detection of breast cancer at early stages is very important and it helps in saving the life of the patient. The mammography is one of the techniques which is used worldwide by radiologists to detect breast cancer. It is very important to detect breast cancer correctly as human life is associated with correct detection. So, nowadays computer-aided diagnose (CAD) systems are used to detect breast cancer from a mammography image. In this work, a transfer learning-based method is used to train the system for the detection of breast cancer. The proposed model is based on a hybrid technique of image enhancement and segmentation for the pre-processing. The pre-processing is done to improve the peak signal to noise ratio (PSNR) value of originally acquired images. The Mammography Image Analysis Society (MIAS) dataset is used in this work along with other clinical images which result in the creation of around 928 images in the dataset.

Aim: This work aims to segment the affected area image from the original image. Then enhanced the segmented image by using PSNR so that better result is helpful to identify the affected area. The correct detection is associated with a life decision.

Result: Mammogram image has been segmented from the affected area. Various type of noises may be induced in the black and white image. The image represents the original, enhanced and segmented image. The PSNR value for an image varies from 33 to 36 . The segmented image is representing the affected regions to limit the image and to eliminate useless details.

Conclusion: This paper put forward the methodology which will segment the affected area from the image. The image has been enhanced based on LCM and CLAHE. Morphology technique has been used along with the Otsu threshold for accurate segmentation. The proposed network is promising as it is trained for mammogram dataset for higher accuracy.

Key Words: Breast cancer, Micro-calcification, Mammogram image, Computer-aided diagnose

\section{INTRODUCTION}

Breast Cancer is one of the most common problems around the whole world, every year lot of women are suffering from breast cancer. Sometimes breast cancer is detected in earlier stages, but sometimes even doctors and radiologists also failed to detect in early stages. ${ }^{1}$ Nowadays most common CAD-based mammogram detection is done to rule out the possibilities of human error. The detection of breast abnormality or cancer is categorized in two possibilities, first is microcalcification which is the beginning stage of cancer and other is mass and microcalcification in which calcium deposits all around the breast region. In this work, a hybrid method proposed for image enhancement and segmentation. The process of image enhancement will start as soon as the system acquires the image. There are several types of unwanted noises induced in the image which may degrade the quality of image . ${ }^{2}$ So, it is very important to remove the noise from images for better segmentation (Figure 1). In this work initially, median filtering is used for noise removal from original acquired image based on 3 x 3 matrix. Then the LCM, A-CLAHE along with wiener filter is used, which completes our hybrid block structure of image enhancement. The enhancement block is used to enhance the PSNR value of the original image. The proposed A-CLAHE method is fundamentally grounded on the adaptive approach for pixel replacement which also reduces the noise from the image. Initially, the images from MIAS dataset are taken which are enhanced to create enhanced images dataset. The enhanced images data set will act as a source of input images for the segmentation block. The

\section{Corresponding Author:}

Kamal Nain Sharma, Research Scholar, IKGPTU, Kapurthala (PB), India. Email: kamalnain3@gmail.com

ISSN: 2231-2196 (Print)

Received: 06.06 .2020
ISSN: $0975-5241$ (Online)

Revised: 23.07 .2020
Accepted: 03.08.2020 
segmentation block is used for extracting features from the image to eliminate useless regions in the image which is done by morphology technique. Also, the Otsu threshold method is applied to image for further enhancement and to remove the unwanted pixels. Further, the regions of the images are segmented based on mass and microcalcification. The proposed work enhances the PSNR of the images than existing methods. ${ }^{3}$

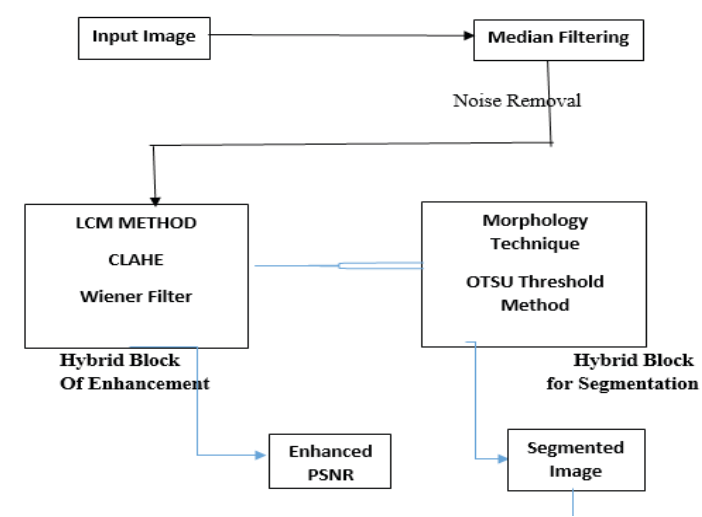

Figure 1: Mammography Image Analysis Methods 1

\section{Literature Review}

Kamra et.al ${ }^{4}$ had proposed an overview of texture classification using an SVM classifier. The proposed method used public domain method Digital Database for Screening Mammogram(DDSM) and MIAS for accuracy $92.34 \%$ in DDSM and $95.34 \%$ is achieved in AD versus non-AD (normal) cases for ground truth ROIs.

Gupta and Tiwari ${ }^{3}$ had used a tool-based method for image enhancement and better segmentation of micro and mass calcification. The author proposed a histogram-based method which helps in enhancing the PSNR value of the inputted image and helps a radiologist to see the image. Also, the segmentation method can segment mass calcification areas from the original image. The average PSNR value obtained by the author proposed method was around 18.90 using CLAHE Median method which enhances PSNR in contrast with other existing methods (Table 2).

Suhail et al. ${ }^{5}$ had classified various mammogram images using a discriminant fisher method. The goal of the paper is to classify the inputted image is benign or malignant. The proposed method encodes the images to a binary value. The linear classification method is used along with the support vector machine to classify the images in both categories and author achieved and accuracy of around 96\%.

Hamidinekoo et al. ${ }^{1}$ author had provided an overview of various recent techniques and methods used in mammography based on deep learning. A variety of techniques and datasets along with methods are reviewed by the author. Most of the present models are based on deep neural network learning with a variety of learning methods like Convolutional Neural Network (CNN), Deep Neural Network(DNN) and recurrent neural network (RNN). The author concludes that most of the existing methods are based on common steps where preprocessing is one of the major steps to improve the original image in the dataset. There are a variety of methods and techniques used by many authors for pre-processing and image enhancement. Then based on any deep learning the network is trained and tested over the images in dataset and finally based on testing and training system can predict or detect the abnormalities in the breast image to classify it in micro or macro calcification. Many existing techniques reviewed by the author had shown the accuracy of up to $85 \%$.

Ragab et al. ${ }^{2}$ had proposed a method to detect breast cancer based on CNN and SVM. The author used a CAD system for image segmentation in which initially goal is to segment the ROI of the image. The CNN method is used to extract features from images, for this purposed author had used Alex net which is a pre-trained model and the last layer of the model is further connected to SVM. DDSM dataset is used in paper and to obtain better accuracy training is done using large data samples. The proposed method used in paper achieved around the accuracy of $77 \%$ with usage of deep convolution network.

Barkalow et al. ${ }^{6}$ had used a deep dual bunch technique for detection of abnormalities in medical images. The proposed method is based on two learning branches, where the first learning branch is used for regional calcification and another branch is used for detection. The effect of classification and localization considering the factor of cost is monitored. The method is tested on a dataset of 543 images with an accuracy of $76 \%$. The further major scope of improvement is suggested by the author to include the phase of learning.

Ouyang et al. ${ }^{7}$ had worked on various HI-SCAN ultrasound images of the breast to classify them in three categories of benign, malignant and normal. Working on images the finding of the author was that in case of more red colour distribution the chance of cancer is high and where in case of blue colour the image is classified as normal category images. The RGB spectrum had shown a major difference between benign and malignant image based on their broadcast colour.

Sannyasi Chakravarthy and Rajaguru ${ }^{8}$ had worked on the noise removal process from the image, and the major goal of the author is to eliminate impulse noise. Initially, the corrupted or noisy pixels are detected and a healing algorithm starts to correct the pixels. The pixels that are easily identified with impulse noise are classified under bidirectional decomposing pixels. The proposed method is compared with various existing methods based on PSNR and PSNR value for the proposed method is higher than existing methods. 
Palee et al. ${ }^{9}$ had proposed a heuristic approach based on DNN learning methods to detect hydatid form mole. The study is done on 17 different critical features to differentiate between PHM and VHM. The characteristic for train the networks are defined as a system extract features out of that to provide neural network data to learn and to classify. The author further suggested to include more images of the cell to explored structure in a better way. Agarwal et al. $2020{ }^{10}$ had developed a method using deep learning for mass detection in the mammogram image. The proposed framework is fully automated for mass detection and used the concept of transfer learning. Overall $78 \%$ accuracy is derived by the author in this paper.

Loizidou et al. ${ }^{11}$ had proposed temporal operations based on automated detection of microcalcification in the breast. In this work, machine learning is combined with temporal subtraction to enhance the accuracy and detection rate. The proposed work is simply based on images operations, where the original and outcome images are compared and further with the help of support vector machine the images are classified into micro or mass calcification.

Sharma and Mehra ${ }^{12}$ had used a combination of two machine learning methods to classify a mammogram image. Initially during preprocessing the region of interest was identified and images were pre-processed using Hue lumen method. Then the network is trained to detect any image as macro calcification with the help of CAD system only.

Huan and Wen ${ }^{13}$ had done face classification with the help of a dual learning model which is based on DNN and transfer learning. The used modes were used to train a network of around 169 layers. The basic classifier used by the author had shown the accuracy of $65 \%$. Transfer learning provides the easiest way to share and understand data.

Bruno et al. ${ }^{14}$ had proposed a novel solution based on scaleinvariant feature transform (SIFT) algorithm and deep learning for spacious regions detection in the mammogram image. Proposed method outperform quite well in contrast with other techniques. The author also states that the lack of dataset availability data is also one of the factors which decrease the accuracy. So, the need is to train the network using bigger datasets to train and test the system properly.

Chowdhary and Mittal ${ }^{15}$ had proposed an efficient method for segmentation and classification of mammogram images using k-mean and SVM learning method. The author used the technique of machine learning to classify the images into various categories. Initially during pre-processing various operations are performed to enhance the PSNR of the image for better classification.

Liu et al. ${ }^{16}$ had proposed a CAD-based technique for cancer segmentation from an image. The proposed method is implemented in MATLAB 2017 using MIAS dataset. The noise removal method is used along with segmentation bitwise, the PSNR value of the final image is higher than the existing methods. The final image had shown sharp edges which make the image quite clear and easy to study.

\section{Proposed Method}

The proposed method of mammogram image enhancement and segmentation is based on two-hybrid blocks. Figure 2 represents the proposed model for enhancement and segmentation.

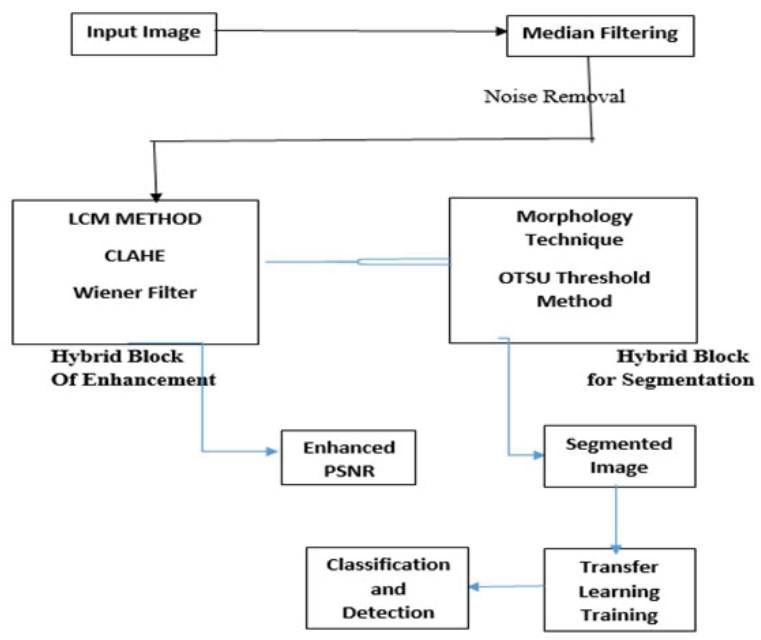

Figure 2: Proposed Methodology (Created for this research).

In this proposed work, dataset MIAS is used along with some other clinical images. Image database had more than 850 Images.

a. Noise Removal: The inputted image is exposed to any type of external noises that may be induced in noise during transfer. These noises will generate an unwanted signal which is very necessary to remove. In this work, 3 x 3 median filtering is used for noise removal.

b. Hybrid Enhancement: The enhancement is done in the inputted image based on LCM, CLAHE and Winner filtering. LCM and CLAHE are histogram-based methods. In LCM method the mean values and standard deviation are computed for an image then the only system will initialize the range for enhancement parameters.

Standard deviation $(\sigma)=\sqrt{\frac{1}{p x p}} \sum_{a=1}^{p-1}(a, b)-m(a, b)$

where pixel $(p)$, image $(f$ and $m)$ and coordinate are $a$ and $b$

Then CLAHE (Contrast Limited Adaptive Histogram Equalization) method is applied after local enhancement is done by LCM. CLAHE method will enhance the contrast 
value for every pixel also reduce unwanted signals. Then wiener filter is used which is best for reduction of MSE (Mean square Error) once the MSE is reduced the original and output image pixels are matched which enhances the PSNR of final output image which is a better image for segmentation.

c. Segmentation: The enhanced image is received from the hybrid block act as the input image for segmentation block. In segmentation block, morphology technique is applied with proposed Otsu threshold. Morphology technique can extract the biological features from an image and helps in better segmentation. Then modified Otsu threshold is used which helps to minimize the variance between black and white pixels and remove unwanted regions from the image. Once the unwanted regions are removed it results in better segmentation.

\section{The algorithm I for A-CLAHE}

\section{Input: MIAS Dataset Images}

Output: Enhance Image

Steps:

1. For $\mathrm{p}=0$ to $\mathrm{p}=12$, image divide into $2 \times 2=4$ small images to 12 × $12=144$ sub images.

2. Initialize empty array to store dynamic entropy $[\mathrm{p}]=$ 0 ;

3. Set $\mathrm{p}=[\mathrm{p}] / /[\mathrm{p}]$ storing maximum value of entropy

4. Do...divide image to sub-images till $\max <=144$.

5. Repeat for each sub-image:

a. Histogram computation for all sub-images

b. Do calculate HPV (Highest Pixel Value) for each sub-image

c. Nominal clip level, range $\mathrm{n}$ to 0 computed. // $\mathrm{n}$ for max value

d. Do repeat for grey level ... loop

e. Histogram Bin $>$ NCL, clip histogram to NCL $n$.

f. Pixels (Histogram $>$ NCL) collected do

g. Random distribute pixels to all histogram for renormalization

h. For all pixels in Interior, Border and Corner regions do sub-images à mapping.

6. Apply above mapping. For each sub-image repeat

7. Final output Image

Algorithm II for Enhancement and Segmentation Combined

Input: Mammographic Image Analysis Society (MIAS) Dataset (D)
Step I: Input I(x, y) $\rightarrow$ loop 3 x 3 window $\forall(\mathrm{x}, \mathrm{y})$, replace $(x, y) \rightarrow\left(x^{\prime} v^{\prime} y^{\prime}\right)$

Step II: Repeat Step I till the end; // Median filtering for noise removal

Step III: Input: I' (x, y) from step 2, A-CLAHE Begin loop

Step IV: Compute peak value for sub-image (s) and nominal clipping, initialize [ ] [ ] for entropy

Step V: Repeat step IV $\forall$ Histogram Bin $>$ Nominal Clip $\rightarrow$ clip to normal else collect exceeded pixels (P)

Step VI: for all $\mathrm{P}$ distribute uniformly $\forall \mathrm{s}$. Output $\rightarrow \mathrm{I}(\mathrm{x}$ ", y") //A-CLAHE

Step VII: I(x ", y") for enhancement local contract method used with wiener filtering

Step VIII: do repeat up to step VII for all I(x,y)

Step IX: Output: Enhanced Dataset (D') with higher peak signal to noise ratio

Step X: Input Dataset (D'), for I(x, y) in D' do

Step XI: I(x, y) $\rightarrow$ Morphology for $\forall \mathrm{I}(\mathrm{x}, \mathrm{y})$ features are extracted for better segmentation

Step XIII: Set Otsu Threshold = Dynamic, compute foreground and background intensity value for all pixels $(\mathrm{x}, \mathrm{y})$

Step XIV: do repeat for all I(x,y) // perform segmentation

Step XV: Output - Segmented Dataset (D") // Contain all segmented Images

d. Proposed Transfer Learning: In this work, we proposed to use a transfer learning method to train and test the network for mammogram images classification. The proposed algorithm is as given below:

\section{Algorithm III: Training Algorithm for Mass and Micro- calcification:}

Input: Segmented Dataset (D")

$\mathrm{L}=$ Length (Segmented Dataset (D")); //Total number of images for training

Mass_images_centroid_list $=\{\} ;$ Centroid of mass image

Normal images centroid list $=\{\}$; Centroid of normal image

Conv5 $\mathrm{x}=512 ; / /$ size of feature window

For $\forall \mathrm{L}(\mathrm{L}=0, \mathrm{~L}<=\mathrm{Max} ; \mathrm{L}++)$ repeat Conv5_x;

Mass_features $=$ construct features $(1$, Conv5 $\mathrm{x}$, Mass $) / / 1$ represent a single image, it will collect all the pixels to be marked by a doctor as cancerous mass.

Normal_features $=$ construct_features $(1$, Normal $) / /$ Conv $5 \_x$ not given as normal image need no learning, collect all pixels not to marks as Mass, Micro 
Micro_features $=$ construct features $(1$, Conv5 $x$, Micro $) / / 1$ represent single image, it will collect all the pixels of Foci LOCs to be marked by doctor small depositions.

net $=$ train_Network(Mass,layers,newFC100) //Mass = Mass data

net $=$ train_Network(Normal,layers,newFC100)// Normal = normal data

net $=$ train_Network(Micro,layers,newFC100) $/ /$ Micro = Micro data

[net,info] = train_Network $\left(\_\right.$_ $) / /$Network Training

Test_preds $=$ classification (net,X_Test, Y_Test,'Mini Batch_Size',mini_Batch_Size);

accuracy $=$ mean(test $\_$preds $==$testImgs.Labels) $* 100 ; / /$ display

end;

\section{RESULTS}

The proposed method is used for the segmentation of a mammogram image. The goal of this work is to segment the affected area from the image. The original mammogram images are black and white. These images are also subjected to various type of noises that may be induced in an image..

The enhanced image is better than the original in terms of clarity and contrast. Figure 3 and 4 represents the original, enhanced and segmented image. The PSNR value for an image varies from 33 to 36 . The segmented image is representing the affected regions to limit the image and to eliminate useless details.
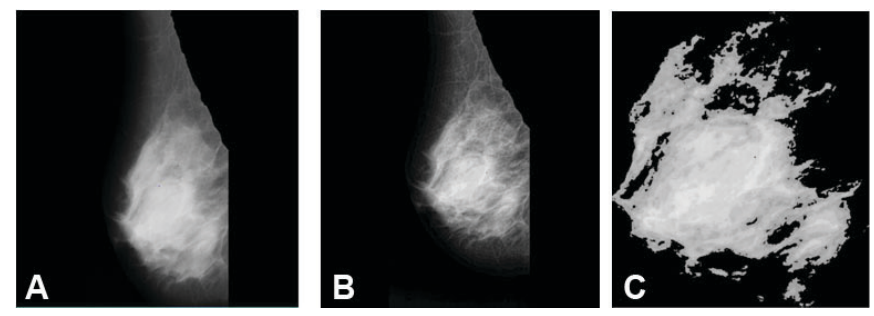

Figure 3: A) Orignal Image; B) Enhanced Image; C) Segmented Image.
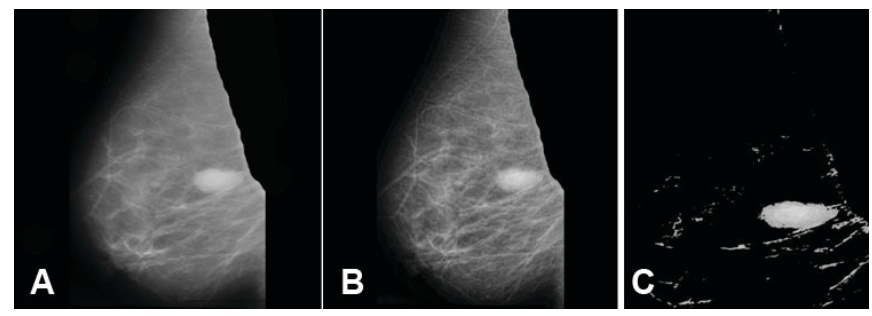

Figure 4: A) Orignal Image; B) Enhanced Image; C) Segmented Image.
The PSNR value of our proposed work is quite acceptable and higher than various existing methods based on CLAHE.

Table I: Value for PSNR [17]

\begin{tabular}{lcccccc} 
MIAS & CLAHE & $\begin{array}{c}\text { HE } \\
\text { IMAGE }\end{array}$ & \multicolumn{5}{c}{ BBHE } & MM- & RMSHE Proposed \\
B & BHE & & \\
mdbo3 & 23.62 & 6.62 & 17.76 & 30.32 & 27.82 & 33.87 \\
mdbo6 & 22.98 & 8.58 & 15.10 & 24.87 & 23.83 & 34.43 \\
mdbo9 & 24.87 & 8.20 & 18.25 & 32.67 & 24.04 & 31.45 \\
mdb10 & 25.76 & 4.30 & 18.76 & 31.90 & 27.14 & 34.67 \\
mdb12 & 24.83 & 9.78 & 18.76 & 32.12 & 26.45 & 33.99 \\
mdb15 & 24.73 & 4.57 & 18.45 & 31.54 & 24.45 & 34.12 \\
\hline
\end{tabular}

\section{DISCUSSION}

The proposed method of the segmentation of the image after removing the noise will provide more accuracy which will further help in the detection of cancer. The differentiation power between the affected region and non-affected region will be increased. Transfer learning has helped in increasing the accuracy on the segmented images.

\section{CONCLUSION}

Breast cancer detection is very important from a mammogram image as life is associated with detection decision. In this paper, a segmentation method had been proposed which can segment the affected areas from the image. As breast cancer is classified as micro and mass calcification, where the calcium deposits may result in cancer. Our proposed method initially enhances the image using a hybrid method based on LCM and CLAHE to enhance the Peak Signal to Noise Ratio (PSNR) value of the original image so that it can be segmented using CAD in a better and accurate way. The segmentation is done using morphology technique along with the Otsu threshold for enhanced and accurate segmentation. The proposed method had shown a quite acceptable PSNR in contrast with various existing algorithms as shown in Table I. Further the segmented images can be used to train the proposed transfer learning network for classification of mammogram images into mass and microcalcification. The proposed training network will be promising as it will be trained specifically for mammogram dataset extracting maximum features for higher accuracy. In future, the segmented image will be feed to a DNN based system to classify the images as micro or mass calcification.

\section{ACKNOWLEDGEMENT}

This work was supported in my research work 


\section{Financial Support: None}

\section{Conflict of interest: None}

\section{REFERENCES}

1. Hamidinekoo A, Denton E, Rampun A, Honnor K, Zwiggelaar R. Deep learning in mammography and breast histology, an overview and future trends. Med Image Anal 2018;47:45-67. https://doi.org/10.1016/j.media.2018.03.006.

2. Ragab DA, Sharks M, Marshall S, Ren J. Breast cancer detection using deep convolutional neural networks and support vector machines. PeerJ 2019;2019. https://doi.org/10.7717/peerj.6201.

3. Gupta B, Tiwari M. A tool-supported approach for brightness preserving contrast enhancement and mass segmentation of mammogram images using histogram modified grey relational analysis. Multidimens Syst Signal Process 2017;28:1549-67. https://doi.org/10.1007/s11045-016-0432-1.

4. Kamra A, Jain VK, Singh S, Mittal S. Characterization of Architectural Distortion in Mammograms Based on Texture Analysis Using Support Vector Machine Classifier with Clinical Evaluation. J Digit Imaging 2016;29:104-14. https://doi.org/10.1007/ s10278-015-9807-3.

5. Suhail Z, Denton ERE, Zwiggelaar R. Classification of microcalcification in mammograms using scalable linear Fisher discriminant analysis. Med Biol Eng Comput 2018;56:1475-85. https://doi.org/10.1007/s11517-017-1774-z.

6. Bakalo R, Goldberger J, Ben-Ari R. Weakly and Semi-Supervised Detection in Medical Imaging via Deep Dual Branch Net 2019.

7. Ouyang Y, Tsui PH, Wu S, Wu W, Zhou Z. Classification of benign and malignant breast tumours using h-scan ultrasound imaging. Diagnostics 2019;9(1): 23-45.https://doi.org/10.3390/ diagnostics9040182.

8. Sannasi Chakravarthy SR, Rajaguru H. Impulse noise removal in mammograms using Bi-dimensional empirical mode decomposition and fast adaptive bilateral filter. Int J Recent Technol Eng 2019;8:674-8. https://doi.org/10.35940/ijrte.B1685.078219.
9. Palee P, Sharp B, Noriega L, Sebire N, Platt C. Heuristic neural network approach in histological sections detection of hydatidiform mole. J Med Imaging 2019;6:1. 451-455. https://doi. org/10.1117/1.jmi.6.4.044501.

10. Agarwal R, Díaz O, Yap MH, Lladó X, Martí R. Deep learning for mass detection in Full Field Digital Mammograms. Comput Biol Med. 2020;12(1):123-129.. https://doi.org/10.1016/j.compbiomed.2020.103774.

11. Loizidou K, Skouroumouni G, Nikolaou C, Pitris C. An Automated Breast Micro-Calcification Detection and Classification Technique Using Temporal Subtraction of Mammograms. IEEE Access 2020;8:52785-95. https://doi.org/10.1109/ACCESS.2020.2980616.

12. Sharma S, Mehra R. Conventional Machine Learning and Deep Learning Approach for Multi-Classification of Breast Cancer Histopathology Images - a Comparative Insight. J Digit Imaging 2020;33:632-54. https://doi.org/10.1007/s10278-019-00307-y

13. Huan EY, Wen GH. Transfer learning with deep convolutional neural network for constitution classification with face image. Multimed Tools Appl. 2020;79:11905-19. https://doi. org/10.1007/s11042-019-08376-5.

14. Bruno A, Ardizzone E, Vitabile S, Midiri M. A Novel Solution Based on Scale Invariant Feature Transform Descriptors and Deep Learning for the Detection of Suspicious Regions in Mammogram Images. J Med Signals Sensors. 2020;10:158-73. https://doi.org/10.4103/jmss.JMSS.

15. Chowdhary CL, Mittal M, Kumaresan P, Pattanaik PA, Marszalek Z. An efficient segmentation and classification system in medical images using intuitionist possibilistic fuzzy Cmean clustering and fuzzy SVM algorithm. Sensors (Switzerland) 2020;20:1-20. https://doi.org/10.3390/s20143903.

16. Liu Q, Liu Z, Yong S, Jia K, Razmjooy N. Computer-aided breast cancer diagnosis based on image segmentation and interval analysis. Automatika. 2020;61:496-506. https://doi.org/10.1 080/00051144.2020.1785784.

17. Akila K, Jayashree LS, Vasuki A. Mammographic image enhancement using indirect contrast enhancement techniques - A comparative study. Procedia Comput Sci. 2015;47:255-61. https://doi.org/10.1016/j.procs.2015.03.205. 